\title{
Sentential Negation Might Share Neurophysiological Mechanisms with Action Inhibition. Evidence from Frontal Theta Rhythm
}

\author{
๑Manuel de Vega, ${ }^{1}$ ○Yurena Morera, ${ }^{1}$ Inmaculada León, ${ }^{1}$ David Beltrán, ${ }^{1}$ Pilar Casado, ${ }^{2}$ and Manuel Martín-Loeches ${ }^{2}$ \\ ${ }^{1}$ Universidad de La Laguna, Campus de Guajara, 38025 La Laguna, Tenerife, Spain, and ${ }^{2}$ Center for Human Evolution and Behavior, Universidad \\ Complutense de Madrid - Instituto de Salud Carlos III, 28029 Madrid, Spain
}

According to the literature, negations such as "not" or "don't" reduce the accessibility in memory of the concepts under their scope. Moreover, negations applied to action contents (e.g., "don't write the letter") impede the activation of motor processes in the brain, inducing "disembodied" representations. These facts provide important information on the behavioral and neural consequences of negations. However, how negations themselves are processed in the brain is still poorly understood. In two electrophysiological experiments, we explored whether sentential negation shares neural mechanisms with action monitoring or inhibition. Human participants read action-related sentences in affirmative or negative form ("now you will cut the bread" vs "now you will not cut the bread") while performing a simultaneous Go/NoGo task. The analysis of the EEG rhythms revealed that theta oscillations were significantly reduced for NoGo trials in the context of negative sentences compared with affirmative sentences. Given the fact that theta oscillations are often considered as neural markers of response inhibition processes, their modulation by negative sentences strongly suggests that negation uses neural resources of response inhibition. We propose a new approach that views the syntactic operator of negation as relying on the neural machinery of high-order action-monitoring processes.

Key words: electrophysiology; embodied cognition; inhibition processes; negation; theta rhythms

\section{Significance Statement}

Previous studies have shown that linguistic negation reduces the accessibility of the negated concepts and suppresses the activation of specific brain regions that operate in affirmative statements. Although these studies focus on the consequences of negation on cognitive and neural processes, the proper neural mechanisms of negation have not yet been explored. In the present EEG study, we tested the hypothesis that negation uses the neural network of action inhibition. Using a Go/NoGo task embedded in a sentence comprehension task, we found that negation in the context of NoGo trials modulates frontal theta rhythm, which is usually considered a signature of action inhibition and control mechanisms.

\section{Introduction}

Negation is a ubiquitous feature of human language. All languages have grammatical markers of negation and children understand and produce some negative statements from the second year of life (Wode, 1977; Austin et al., 2014). Behavioral studies

Received 0ct. 12, 2015; revised April 14, 2016; accepted April 16, 2016.

Author contributions: M.d.V., I.L., P.C., and M.M.-L. designed research; M.d.V., Y.M., and D.B. performed research; Y.M., I.L., D.B., and M.M.-L. analyzed data; M.d.V., D.B., and M.M.-L. wrote the paper.

This work was funded by the Spanish MINECO (Grants SEJ2011-28679 and PSI2015-66277-R to M.d.V. and Grant PSI2013-43107-P to M.M.-L.), the Campus de Excelencia Tricontinental, and the European Regional Development Funds.

The authors declare no competing financial interests.

Correspondence should be addressed to Manuel de Vega, Departamento de Psicología Cognitiva, Universidad de La Laguna, Campus de Guajara, 38205 La Laguna, Santa Cruz de Tenerife, Spain. E-mail: mdevega@ull.es.

DOI:10.1523/JNEUROSCI.3736-15.2016

Copyright $\odot 2016$ the authors $\quad 0270-6474 / 16 / 366002-09 \$ 15.00 / 0$ indicate that negative sentences sometimes demand additional cognitive resources in comparison with affirmative sentences (Clark and Chase, 1972; Carpenter and Just, 1975; Kaup, 2006) and the presence of negation in a sentence immediately reduces the level of accessibility of the negated concept (MacDonald and Just, 1989; Kaup, 2001; Kaup and Zwaan, 2003).

Neuroimaging studies have reported that negative actionrelated sentences generally reduce the activation of motor and premotor cortex in comparison with affirmative action sentences (Tettamanti et al., 2008; Tomasino et al., 2010). Moreover, paired-pulse transcranial magnetic stimulation applied over the right motor cortex reduced cortico-spinal excitability while participants read affirmative action sentences, but not while they read negative action sentences, indicating that only understanding the former recruits motor networks (Liuzza et al., 2011). Behavioral measures obtained with sentence-action interference 
paradigms also showed reduced involvement of the motor cortex in negated action sentences (Aravena et al., 2012; Bartoli et al., 2013).

Most studies reviewed up to now have proved useful in analyzing the impact of negation on the cognitive (and neural) representations of sentence meaning, consistently showing that negation reduces the strength of brain activations in specific cortices. However, negation remains itself an unexplained neural black box. In this study, we used a new research strategy aimed at exploring the neural mechanism of negation itself. We propose the hypothesis that understanding sentential negation relies partially on the neurophysiological mechanisms of response inhibition. To motivate this hypothesis, let us consider these premises: (1) negation seems to reduce or suppress the accessibility of previously activated representations and (2) response inhibition consists of suppressing previously activated responses and also operates to suppress previously activated representations (Nakata et al., 2008; Smith et al., 2008). Therefore, we may expect that the general mechanisms of behavior inhibition or control are involved in the proper understanding of negation.

Response inhibition consists of cancelling planned actions or suppressing alternative behaviors or representations that are inappropriate, unsafe, or no longer required (Chambers et al., 2009). One representative experimental paradigm to analyze response inhibition is the classical Go/NoGo task, in which Go trials induce a prepotent response, whereas NoGo trials require a response suppression or inhibition. Electrophysiological studies have shown enhanced $\mathrm{N} 2$ and P3 components of the ERP over frontocentral electrodes as signatures of response inhibition (Bokura et al., 2001; Smith et al., 2008; Maguire et al., 2009). Moreover, time-frequency analysis of the EEG signal usually obtains power enhancement in the theta band $(4-7 \mathrm{~Hz})$ over frontocentral sites and sometimes in the delta band (1-3 Hz) over centro-parietal sites for the NoGo condition (Nigbur et al., 2011; Huster et al., 2013; Cohen, 2014; Harper et al., 2014), probably indexing conflict detection and response evaluation processes, respectively (Huster et al., 2013; Harper et al., 2014).

As stated previously, the purpose of the present study was to test whether the comprehension of sentential negation uses the brain mechanisms underlying response inhibition. To this end, participants were asked to understand sentences with affirmative or negative polarity (Table 1 ) embedded in a Go/NoGo task. To minimize the participants' use of special strategies to deal with these two temporally overlapping tasks, their performance was tested independently; namely, the Go/NoGo task relied on a standard visual cue (color circle) presented online, whereas the language task was eventually measured for comprehension after finishing the whole trial by answering a control question. The

\section{Table 1. Examples of experimental and filler sentences (with literal translations} into English in parentheses)

\begin{tabular}{l}
\hline Experimental sentences \\
Affirmative: Ahora sí cortarás el pan (Now you will [yes] cut the bread) \\
Negative: Ahora no cortarás el pan (Now you will not cut the bread) \\
Possible control questions \\
Ahora sí cortarás el pan (Now you will [yes] cut the bread) \\
Ahora no cortarás el pan (Now you will not cut the bread) \\
Ahora sí comprarás el pan (Now you will [yes] buy the bread) \\
Ahora no cortarás el queso (Now you will not cut the cheese) \\
Filler sentences \\
Affirmative: Después sí llamarás al taxi (Afterwards you will [yes] call the taxi) \\
Negative: Luego no comprarás las flores (Thereafter you will not buy the flowers)
\end{tabular}

A control question could follow the experimental sentences in one of four versions: correct for affirmative sentences, correct for negative sentences, incorrect verb, or incorrect noun. rationale of this double-task study is as follows: if negation shares neural processes with response inhibition, then we may expect interactions between the Go/NoGo task and the sentence polarity on the EEG markers of response inhibition. Specifically, we hypothesize that theta/delta oscillations, the power of which increases in the NoGo condition, will be modulated by negative sentences. We can also predict that the ERP waves N2 and P3, which are enhanced in the NoGo condition, will be modulated by the context of negative sentences. Given the novelty of the experimental paradigm used here and the potential theoretical relevance of the results, we decided to perform two experiments to ensure that the effects were replicable.

\section{Materials and Methods}

Participants. A total of 57 psychology undergraduate students participated in two experiments: 33 in Experiment 1 ( 28 females; mean age 21 years old, range 18-42) and 24 in Experiment 2 (17 females; mean age 20 years; range 18-28). All participants gave informed consent and received course credit for their participation. All were neurologically healthy, right-handed native Spanish speakers and had normal or corrected-tonormal eyesight. Four participants in Experiment 1 and one participant in Experiment 2 were removed from the analysis because of an excessive number of ocular artifacts.

Design and materials. Design and materials were identical in the two experiments. A two cue $(\mathrm{Go} / \mathrm{NoGo}) \times$ two sentence polarity (affirmative/negative) repeated-measures experimental design was used. A total of 266 experimental sentences were constructed in Spanish, each in two versions: affirmative and negative. In addition, 40 filler sentences were included that differed from the experimental ones in the first word only: all of the experimental sentences started with "ahora" (now), whereas the fillers used the adverbs "después" (afterward) or "luego" (thereafter). Examples of the materials are shown in Table 1.

To maintain participants' attention on the task, $\sim 33 \%$ of the sentences were followed by a yes/no recognition question consisting of either a sentence that was identical to the previous sentence ("yes" response) or a modified version in which the polarity, verb, or noun had been changed ("no" response). The experimental sentences were divided into a set of 186 Go trials (70\%) and another set of 80 NoGo trials (30\%), which were controlled for lexical factors of the verb and noun, as shown in Table 2. The number of words in the experimental sentences was also controlled using the Spanish emphatic affirmative "sí" to parallel the presence of the negation operator (Table 1).

Procedure. After receiving instructions participants were given 15 practice trials, followed by three blocks of experimental and filler trials presented in random order. Two of these blocks consisted of 101 trials, with 88 experimental sentences ( 44 affirmative +44 negative) and 13 filler sentences ( 7 affirmative +6 negative); the other block consisted of 104 trials, with 90 experimental sentences ( 45 affirmative +45 negative) and 14 filler sentences ( 7 affirmative +7 negative). In each block, $70 \%$ of trials included a Go cue and the remaining received a NoGo cue. Blocks were ordered randomly for each participant; within each block, trials were presented randomly.

Each trial consisted of a sentence presented, one word at a time, on a 24 inch monitor, as well as the Go or NoGo cue. All events in a trial were controlled by means of E-prime software (version 2.1; Psychology Software Tools) according to the temporal sequences shown in Figure 1. Three-hundred milliseconds after the verb onset, the Go/NoGo cue ap-

Table 2. Mean scores of lexical frequency, length (number of letters), and imageability of the verb and the noun in Go and NoGo trials

\begin{tabular}{llllll}
\hline & Verb & & & Noun \\
\cline { 2 - 3 } \cline { 6 - 6 } & Go & NoGo & & Go & NoGo \\
\hline Frequency & 1.03 & 1.06 & & 0.99 & 1.04 \\
Length & 6.13 & 6.14 & & 6.2 & 5.39 \\
Imageability & 3.77 & 3.82 & & 3.80 & 3.96
\end{tabular}

Statistical testing did not find any significant difference between $\mathrm{Go}$ and NoGo homologous words $(t<1)$. 
peared above the word as a yellow or blue circle, respectively, remaining on screen for 300 ms. In trials with a recognition task (33\%), a question mark was depicted $300 \mathrm{~ms}$. preceding the probe sentence. The trials were identical in both experiments except that the verb presentation time was shorter in Experiment 2 (700 $\mathrm{ms})$ than in Experiment 1 (1200 ms), as Figure 1 illustrates. With the faster presentation time of the critical events in Experiment 2, we intended to introduce more urgency in the Go/ NoGo response, increasing the difficulty of the decision and eventually increasing the number of commission and/or omission errors.

Go responses were given by pressing, with the right index finger, a key labeled with a yellow sticker (corresponding to the letter "L" on the keyboard) and responses to the recognition task consisted of pressing, with either the index or middle finger of the left hand, one of two keys labeled as "yes" or "no" (corresponding to the " 1 " and " 2 " keys on the keyboard, respectively). The recognition sentences were correct in $50 \%$ of trials. Correct response reaction times and accuracy data were collected both for the Go/NoGo task and for the recognition task.

EEG recording and preprocessing. EEG and EOG signals were recorded using $\mathrm{Ag} / \mathrm{AgCl}$ electrodes mounted in elastic Quick-caps (Compumedics). EOG signal was measured from two bipolar channels: one from two electrodes placed at the outer canthus of each eye and the other from two electrodes above and below the left eye. EEG signal was recorded from 60 electrodes arranged according to the standard 10-20 system, with additional electrodes placed at $\mathrm{cb} 1 / \mathrm{cb} 2$ and also on the left and right mastoids (M1/M2). All EEG electrodes were referenced online to an electrode at vertex and re-referenced offline to linked mastoids. EEG and EOG signals were amplified at $500 \mathrm{~Hz}$ sampling rate using Synamp2 amplifier (Neuroscan; Compumedics), with high- and low-pass filters set at 0.05 and $100 \mathrm{~Hz}$, respectively. EEG electrode impedance was kept at $<5 \mathrm{k} \Omega$.

EEG data preprocessing and analysis were conducted using Fieldtrip Toolbox (Oostenveld et al., 2011). Epochs were extracted from $2.5 \mathrm{~s}$ precue ( $\mathrm{Go} / \mathrm{NoGo}$ signal) onset to $2.5 \mathrm{~s}$ postcue onset, resulting in 5000 ms epochs. Trials with drifting or large movement artifacts were removed by visual inspection before analysis. Independent component analysis was applied to the data to remove the effects of blinks and eye movements. Remaining trials with EEG voltages exceeding $70 \mu \mathrm{V}$ measured from peak to peak at any channel were also removed.

$E R P$, time-frequency analyses, and source estimation. To compute the ERPs, artifact-free EEG segments were averaged separately for each of the four experimental conditions using as baseline the $200 \mathrm{~ms}$ period preceding the Go/NoGo cue. The resulting ERP waveforms were evaluated statistically using the cluster-based random permutation method implemented in Fieldtrip (Maris and Oostenveld, 2007). This method deals with the multiple comparisons in space and time by identifying, over the whole ERP segment (here, 30,000 sample points: 500 time points and 60 channels), clusters of significant differences between conditions (sample points in close spatial and temporal proximity) while effectively controlling for type 1 error.

This statistical approach allows only for pairwise comparisons. Therefore, certain prior calculations were performed to evaluate the main effects and the interaction of the current $2 \times 2$ design. Regarding the main effects, an average of the two conditions corresponding to the same level of each of the two factors was calculated for each subject and comparisons were performed using these two averages. For example, for the main effect of Cue, the average Go-affirmative and Go-negative trials formed the level "Go" of the factor and was compared with the one calculated for the level "NoGo" (average NoGo-affirmative and NoGo-negative). For testing the interaction, affirmative minus negative difference waveforms were computed for each cue separately and then these difference waveforms were compared statistically.

For the computation of the time-frequency representation (TFR) in Experiment 1, spectral power $(1-30 \mathrm{~Hz})$ was obtained by convolving 6-cycle complex Morlet wavelets with each single-trial EEG epoch. The resulting EEG power representations were normalized by subtracting, in a frequency fashion, the baseline from the power in every time point and dividing this difference by the baseline mean power. The $500 \mathrm{~ms}$ preceding the onset of the polarity word (affirmative "sí," negative "no") was used as the baseline, which means that resulting TFRs reflect power changes relative to this period. Finally, before the statistical analysis, the single-trial TFRs were averaged separately for each of the four experimental conditions. The cluster-based random permutation method was also applied for the statistical analysis of averaged TFRs. With respect to the approach followed to analyze ERPs, the only difference was the size of the data matrix used as input, which included for TFRs 60 channels, 100 time points $(0-1 \mathrm{~s}$ after cue onset in $10 \mathrm{~ms}$ steps), and 15 frequencies (1-30 Hz in $2 \mathrm{~Hz}$ steps). The same statistical approach was used to analyze TFRs in Experiment 2 except that the TFR data matrix used as input for the permutation method did not include the whole range of frequencies used in Experiment 1. Instead, we defined delta and theta bands in advance (2-3 and $4-7 \mathrm{~Hz}$, respectively) and averaged the data along these two bands. This data-reduction strategy was motivated by the hypothesis-driven, confirmatory purpose of Experiment 2.

In Experiment 1, to obtain a glimpse of the brain regions involved in the scalp delta/theta main effect of cue, we estimated the neural sources for the difference between NoGo and Go trials using the beamformer spatial filtering technique DICS (Dynamical Imaging of Coherent Sources; Gross et al., 2001), as implemented in Fieldtrip Toolbox. More specifically, we calculated, first, the cross-spectral density matrix from the data for the time interval between 200 and $600 \mathrm{~ms}$ after the Go/NoGo cue onset using sleeping tapers to obtain $\pm 3 \mathrm{~Hz}$ spectral smoothing around the $4 \mathrm{~Hz}$ center frequency. This yielded an estimate of power activity in the $1-7 \mathrm{~Hz}$ frequency band. Next, we used the MIN template-based forward model included in Fieldtrip (standard boundary element method-based model; see Oostenveld et al., 2011 for details) to construct the leadfield matrix for our specific set of EEG electrodes, by discretizing the forward model into $5 \mathrm{~mm}$ resolution grids. This leadfield matrix was further combined with the cross-spectral density estimates to compute adaptive spatial filters for each specific brain grid location using a $6 \%$ regularization parameter (lambda). Note that we computed first common spatial filters for all trials and then projected separately the resulting filters to the frequency estimates for each condition. In addition, before 
computing spatial filters, we forced the leadfield to project onto the orientation of maximal power, which means that, for each grid, only a single beamformer value was computed instead of a vector of values reflecting the weight for each grid direction. As for the sensor-level analyses, the same computations were performed on the baseline period (500 $\mathrm{ms}$ preceding the onset of the affirmative/negative word), the power estimate of which was used to calculate the relative power increase in the posttrial cue interval for each condition.

Finally, in Experiment 2, to test whether the EEG time-frequency signal was related to trial-varying performance, within-subject singletrial correlations between EEG time-frequency signal and reaction time were conducted on Go trials. The procedure for this correlational analysis was as follows. First, for each participant, data at every channel, frequency, and time point were correlated with reaction time over trials using Spearman correlations. This produced a time-frequency map of correlation coefficients for each participant. Next, statistical significance was computed, at the group-level, on Fisher's $z$-transformed correlation values resulting from averaging coefficient across the electrodes for the frequency and time bin of interest, namely those resulting from the previously obtained cue $\times$ polarity interaction.

\section{Results}

\section{Behavioral results}

Go-NoGo task

Go-trial reaction times in milliseconds were analyzed after removing outliers with scores three SDs above the participant's mean $(\sim 1 \%$ of trials in Experiment 1 and $1.2 \%$ of trials in Experiment 2). In Experiment 1, sentence polarity did not produce significant effects on reaction times (affirmative: $M=357$, $\mathrm{SD}=52.5$; negative: $\mathrm{M}=359, \mathrm{SD}=48.6 ; t_{(28)}=0.064, p=$ $0.524)$. Polarity conditions did not differ either on commission errors in NoGo trials (affirmative: $\mathrm{M}=2.2 \%, \mathrm{SD}=3.4 \%$; negative: $\mathrm{M}=2.1 \%$; $\left.\mathrm{SD}=3.1 \% ; t_{(28)}=0.107, p=0.916\right)$ or on omission errors in Go trials (affirmative: $\mathrm{M}=12 \%, \mathrm{SD}=$ $6.4 \%$; negative: $\mathrm{M}=12 \% ; \mathrm{SD}=6.7 \% ; t_{(28)}=0.144, p=$ $0.887)$. Notice that the number of omission errors in Experiment 1 was unexpectedly high ( $\sim 12 \%$ of trials). After the data collection, we realized that the key assigned to Go responses sometimes failed to register them and send the corresponding triggers to the EEG. This failure occurred randomly (unbiased across polarity conditions) and it was therefore appropriate to run the EEG analyses on the remaining valid trials. The likelihood that these key failures resulted in unregistered commission errors in NoGo trials is negligible.

In Experiment 2, the response key problem was corrected and the rate of omission errors in Go trials was very low $(\sim 1 \%)$, contrasting with the artifactual high rate of omission errors obtained in Experiment 1. Beyond that, the pattern of results in Experiment 2 was very similar to the pattern obtained in Experiment 1 . First, Polarity conditions did not differ either on commission errors in NoGo trials (affirmative: $\mathrm{M}=2.2 \%, \mathrm{SD}=$ $3.4 \%$; negative: $\mathrm{M}=2.1 \%$; $\mathrm{SD}=3.1 \%$; $\left.t_{(23)}=0.81, p=0.43\right)$ or on omission errors in Go trials (affirmative: $\mathrm{M}=1 \%, \mathrm{SD}=1.1 \%$; negative: $\left.\mathrm{M}=1 \% ; \mathrm{SD}=1.5 \% ; t_{(23)}=0.30, p=0.76\right)$. However, there was a main effect of cue; namely, commission errors were more frequent in NoGo trials than omission errors in Go trials $\left(F_{(1,23)}=4.324, p=0.049, \eta_{\mathrm{p}}^{2}=0.158\right)$. Second, as in Experiment 1 , sentence polarity did not produce any significant effect on Go reaction times (affirmative: $\mathrm{M}=357, \mathrm{SD}=42$; negative: $\mathrm{M}=$ $\left.359, \mathrm{SD}=43 ; t_{(23)}=0.84, p=0.41\right)$. Moreover, we performed an experiment $\times$ polarity ANOVA on Go trials reaction times and we found similar results in both studies (in all cases: $F_{(1,55)}<1$ ), indicating that the response key problem in Experiment 1 did not affect the general pattern of the chronometric data. Finally, an experiment $\times$ polarity ANOVA on errors in NoGo trials con-
Table 3. Performance in the recognition task in Experiments 1 and 2

\begin{tabular}{lcrrrr}
\hline & \multicolumn{3}{l}{ Sentence polarity } & & \\
\cline { 2 - 3 } & \multicolumn{1}{l}{ Experiment 1 } & & & Experiment 2 & \\
\cline { 2 - 3 } \cline { 5 - 6 } & Affirmative & Negative & & Affirmative & Negative \\
\hline Go & $1388(304)$ & $1433(320)$ & & $1464(296)$ & $1493(288)$ \\
RT & $4.9(3.9)$ & $5.7(4.8)$ & & $3.0(4.0)$ & $5.4(7.0)$ \\
Errors & & & & \\
NoGo & $1491(352)$ & $1485(356)$ & & $1529(340)$ & $1568(303)$ \\
RT & $6.3(8.2)$ & $6.9(7.1)$ & & $5.5(6.8)$ & $5.5(6.0)$ \\
Errors & & &
\end{tabular}

Mean reaction times (RTs) in milliseconds and percentage of errors as a function of cue ( $\mathrm{Go} / \mathrm{NoGO})$ and polarity (affirmative/negative). SDs are shown in parentheses.

firmed a similar rate of commission errors in Experiment 1 and Experiment $2\left(\sim 2.2 \% ; F_{(1,55)}<1\right)$, supporting the idea that the amount of unregistered commission errors in Experiment 1 was negligible.

\section{Recognition task}

Table 3 shows performance on the recognition test for both experiments. In Experiment 1, there was a main effect of cue, with responses faster in the Go than in the NoGo trials $\left(F_{(1,28)}=\right.$ $\left.24.356, p=0.0001, \eta_{\mathrm{p}}^{2}=0.465\right)$. In addition, the cue $\times$ polarity interaction was significant $\left(F_{(1,28)}=4.349, p=0.046, \eta_{\mathrm{p}}^{2}=\right.$ $0.134)$. Specifically, affirmative sentences produced faster responses than negative sentences for Go trials $\left(t_{(28)}=3.139, p=\right.$ $0.004)$, whereas affirmative and negative sentences did not differ for NoGo trials $\left(t_{(28)}=0.229, p=0.820\right)$. Concerning Experiment 2 , responses were faster $\left(F_{(1,23)}=27.888, p=0.0001, \eta_{\mathrm{p}}^{2}=\right.$ $0.548)$ and more accurate $\left(F_{(1,23)}=4.389, p=0.047, \eta_{p}^{2}=0.160\right)$ in Go than in NoGo trials. There was also a main effect of polarity on accuracy: larger number of errors in negative than in affirmative sentences $\left(F_{(1,23)}=9.478, p=0.005, \eta_{\mathrm{p}}^{2}=0.292\right)$. Finally, the cue $\times$ polarity interaction was significant on errors $\left(F_{(1,23)}=\right.$ 5.86, $\left.p=0.024, \eta_{\mathrm{p}}^{2}=0.203\right)$. Specifically, for Go trials, participants produced more errors in negative than affirmative sentences $\left(t_{(23)}=4.187, p=0.0001\right)$, whereas for NoGo trials, affirmative and negative sentences did not differ $\left(t_{(23)}=0.189\right.$, $p=0.852)$.

\section{ERP results}

In Experiment 1, statistical analyses of ERPs for the contrast between NoGo and Go trials obtained significant clusters; however, neither the effect of sentence polarity nor the interaction between polarity and cue yielded significant effects. As Fig. 2 illustrates, in Experiment 1, NoGo trials elicited larger negative amplitudes than Go trials between 232 and $416 \mathrm{~ms}$ after the Go/NoGo cue onset $\left(T_{\text {(maxsum) }}=2580.5, p<0.001\right)$. The timing of this effect, along with the direction of the differences, were consistent with the extensively reported enhanced N2 activity for NoGo conditions (Bokura et al., 2001; Bruin and Wijers, 2002; Maguire et al., 2009). In addition, at a later time interval (420-680 ms), NoGo trials showed larger positive amplitudes than Go trials in frontocentral sites $\left(T_{(\text {maxsum })}=1598.3, p<0.001\right)$, thereby replicating the frontocentral distribution of $\mathrm{P} 3$ typically obtained in Go/ NoGo paradigms.

In Experiment 2, again, only the comparison between NoGo and Go trials yielded significant effects on the ERPs $\left(T_{(\text {maxsum })}=5072.2, p<0.001 ; 240-415 \mathrm{~ms}\right)$. The direction and topography of the effects were consistent with the frontocentral P3 component described in Experiment 1 (see Fig. 5A). The time segment corresponding to the N2 component did 
A

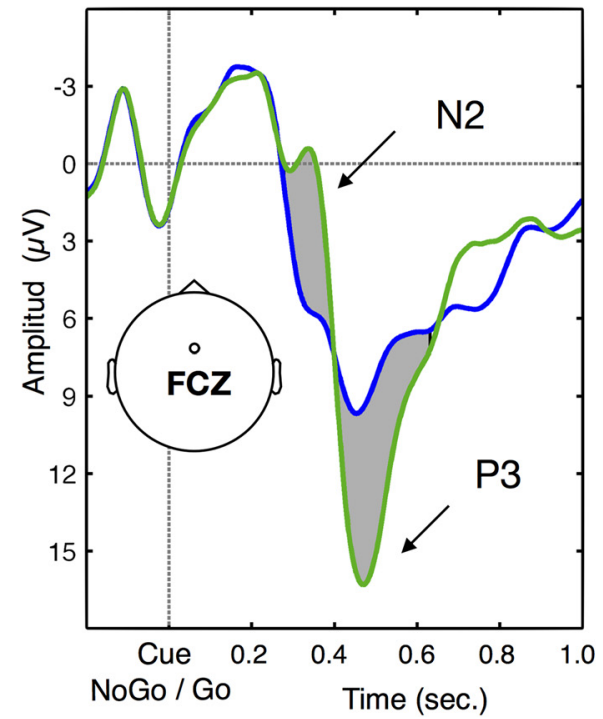

B
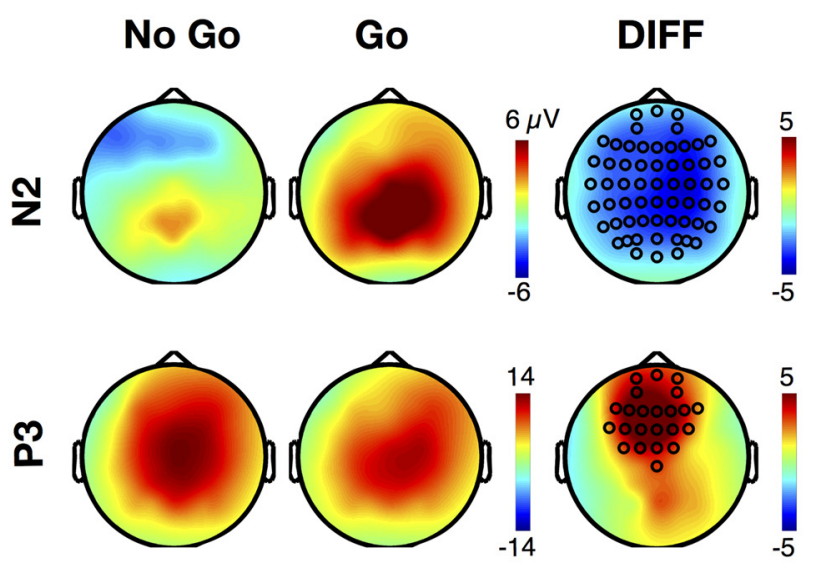

No Go $=$ Go

Figure 2. Experiment 1. Event-related potentials. A, Waveforms of the significant main effects of cue (NoGo vs Go) on the N2 and P3 time windows identified by cluster-based random permutation analysis and shown here as gray-shaded areas in one representative electrode (FCz). B, Scalp distribution of the ERP activity in the identified time windows for NoGo and Go trials. $\boldsymbol{C}$, Difference between NoGo and Go trials in the two significant time windows (the small circles correspond to the electrodes with significant differences).

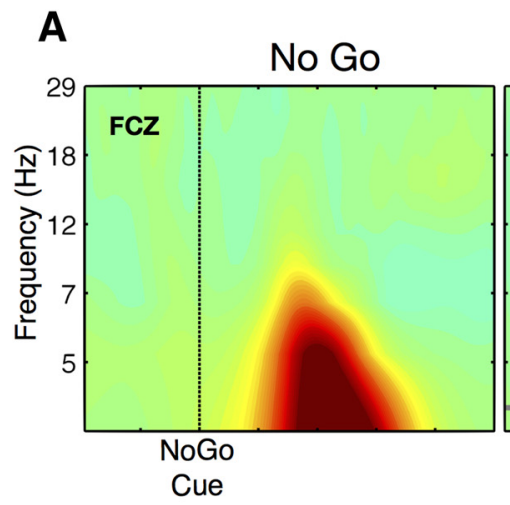

B
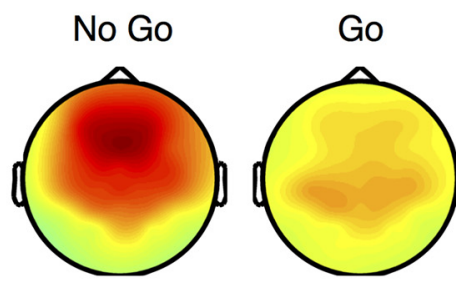

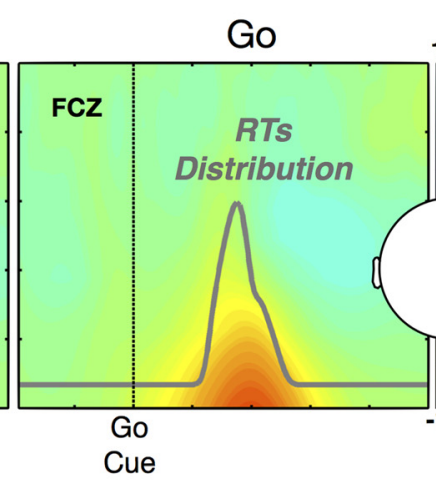

C

Time: $\mathbf{2 0 0 - 5 0 0 ~ m s ~ F r e q u e n c y : ~ 1 - 7 ~ H z ~}$

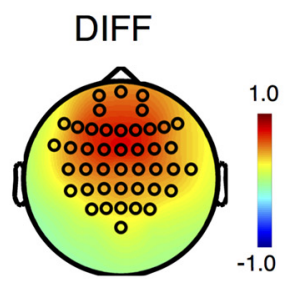

$-1.0$

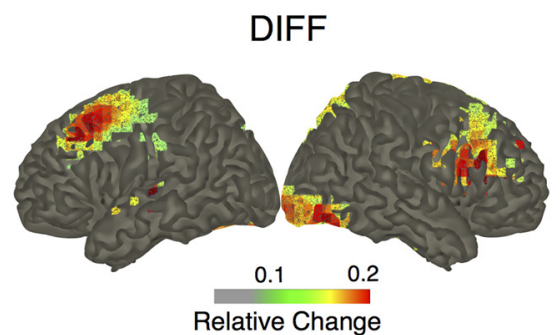

Relative Change

Figure 3. Experiment 1. Time-frequency analysis of the cue main effects in the delta/theta frequency bands. A, Main effect of the NoGo and Go trials and cluster of significant NoGo-Go differences shown in a representative electrode (FCz). B, Scalp distribution of the effects, corresponding to the significant NoGo-Go differences (the small circles correspond to the electrodes with significant differences). $\boldsymbol{C}$, Source estimation of the NoGo-Go contrast. Significant clusters were obtained in the left premotor cortex, the right inferior frontal gyrus, and the right occipital lobe.

not yield significant differences; nonetheless, there was a tendency toward NoGo trials eliciting larger N2 amplitudes than Go trials at frontocentral sites.

\section{Time-frequency results}

The analyses of the TFR revealed a main effect of Cue in Experiment 1 (Fig. 3). Consistent with prior research with the Go/NoGo paradigm, NoGo trials showed stronger power increase than Go trials in a cluster of frontocentral sites
$\left(T_{(\text {maxsum })}=7016.3, p<0.001\right)$. The time interval of maximal delta and theta-band differences between NoGo and Go trials approximately matches the participants' reaction time distribution for Go trials, suggesting that the modulation of delta/ theta oscillations is closely related to the process involved in refraining a prepotent Go response (Fig. 3A). Although broadly extended in time, space, and frequency, the significant cluster showed maximal differences between 200 and $500 \mathrm{~ms}$ after the Go/NoGo cue onset, mainly over frontocentral scalp 
A

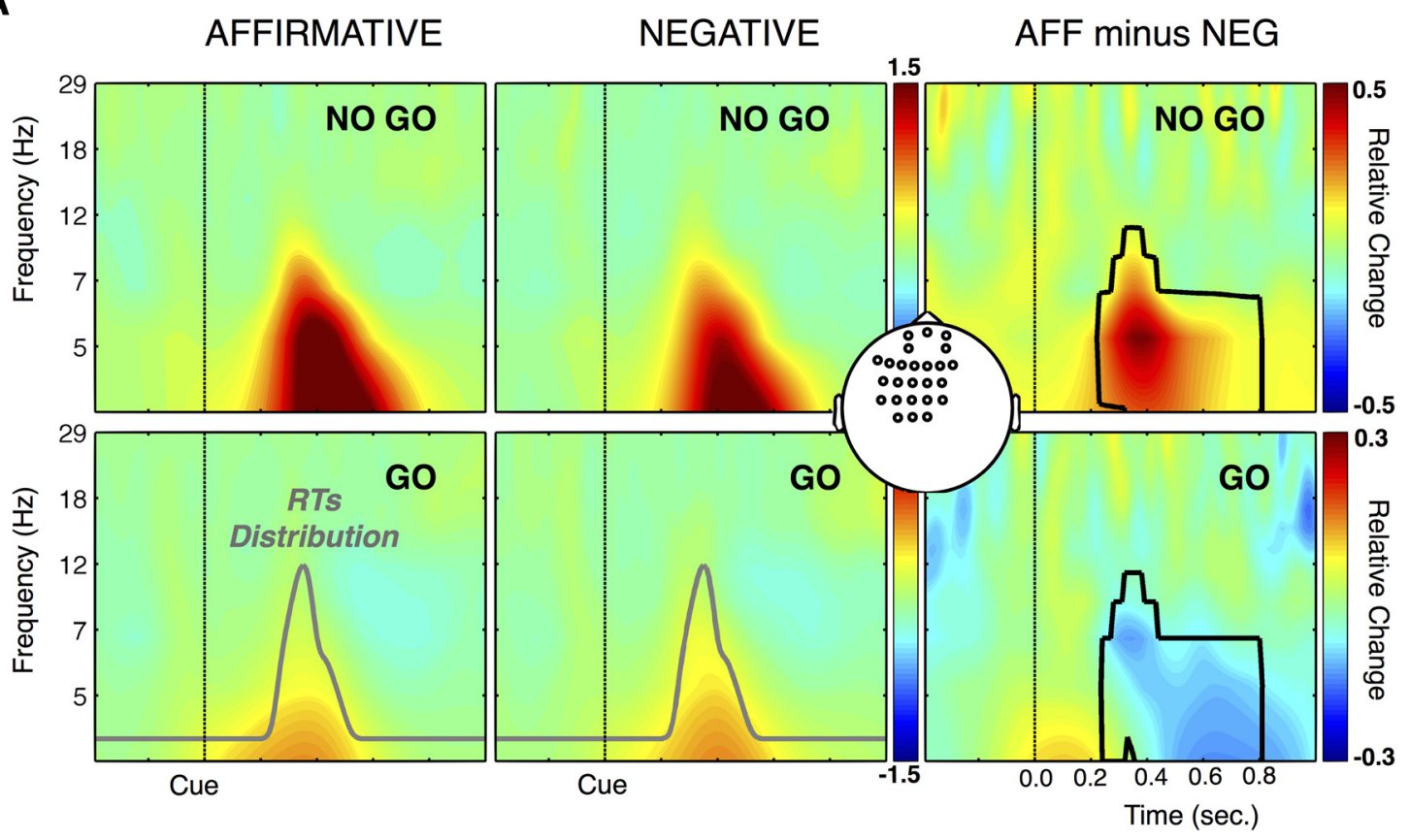

B

Time: $\mathbf{2 0 0 - 4 5 0 ~ m s ~ T h e t a : ~} \mathbf{4 - 7} \mathbf{~ H z}$

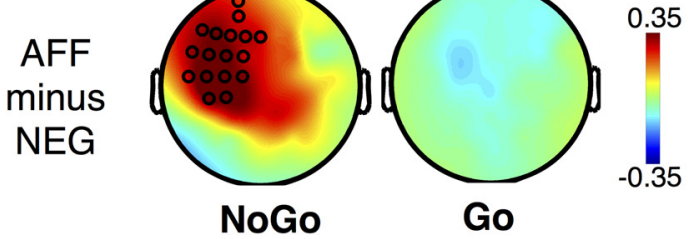

Time: $\mathbf{4 5 0 - 8 0 0 ~ m s ~ D e l t a : ~} \mathbf{2 - 3} \mathbf{~ H z}$

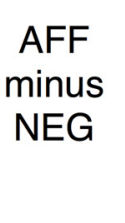

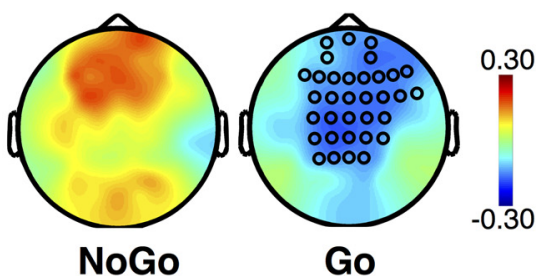

Figure 4. Experiment 1. Time-frequency analysis of the cue $\times$ polarity interaction. $\boldsymbol{A}$, Average time-frequency power in the whole set of significant electrodes (signaled in the inserted white map): Affirmative-NoGo trials elicited larger theta power than negative-NoGo trials in a relatively early interval associated with response inhibition, whereas affirmative-Go trials elicited less delta power than negative-Go trials in a later (postresponse) interval. $\boldsymbol{B}$, Distribution on the scalp of both the early theta-band modulation and the later delta-band modulation (the small circles correspond to the electrodes with significant differences).

sites and for a frequency range of delta and theta $(1-7 \mathrm{~Hz})$ oscillations (Fig. $3 B$ ). Moreover, the source estimation of the time-frequency differences between NoGo and Go trials shows clusters of activation localized in the left premotor cortex, the right inferior frontal gyrus, and the right occipital lobe, with all of these regions reflecting larger power increases for NoGo than for Go trials (Fig. 3C).

Most importantly, in Experiment 1, the cue $\times$ polarity analysis obtained a significant interactive cluster in the $1-8 \mathrm{~Hz}$ frequency band, extending between 200 and $800 \mathrm{~ms}$ after the Go/NoGo cue onset and with a frontocentral distribution $\left(T_{\text {(maxsum) }}=1017.1, p<0.01\right)$. This interactive cluster seemed to include effects of sentence polarity that varied in latency and frequency as a function of cue (Fig. 4). In particular, the results shown in Figure $4 A$ suggest that, in NoGo trials, theta power increased more for affirmative than for negative sentences at a relatively early time interval, which again approximately matched the participants' reaction time distribution for Go trials. In contrast, in Go trials, delta power increased less for affirmative than for negative sentences in a later time interval. To characterize these two apparently distinct effects of sentence polarity statistically, we computed averaged relative power for two frequency bands (theta: $4-7 \mathrm{~Hz}$ and delta: 1-3 $\mathrm{Hz})$ and two time intervals (200-450 ms and 450-800 ms) and conducted comparisons between affirmatives and negatives in each cue (NoGo and Go). For the earlier time interval, affirmative-NoGo trials elicited larger theta power than negative-NoGo trials over left frontal and central sites $(p<$ 0.01), whereas there was no effect in the delta band (Fig. $4 B$ ). In contrast, for the later time interval, affirmative-Go trials produced smaller delta power than negative-Go trials over frontal and central sites $(p<0.025)$, with the difference showing a slightly right-lateralized topographical distribution.

In Experiment 2, the cue $\times$ polarity analysis in the theta band $(4-7 \mathrm{~Hz})$ identified a significant cluster between 200 and $550 \mathrm{~ms}$ after the Go/NoGo cue onset $\left(T_{\text {(maxsum) }}=798.5, p<0.025\right)$. As for Experiment 1, this cluster arose around the time of response, showing chiefly a frontocentral distribution. Further comparisons on the values resulting from averaging across the time and sites belonging to the significant cluster revealed that, for NoGo trials, theta power increased more for affirmative than negative sentences $(p<0.01)$, whereas the opposite pattern was true for the Go trials $(p=0.07)$. In addition, these follow-up comparisons also showed the presence of a strong effect of cue. Despite the interaction effect, NoGo trials elicited stronger theta power increases than Go trials for both affirmative $(p<0.0001)$ and negative sentences $(p<0.05)$. 
A

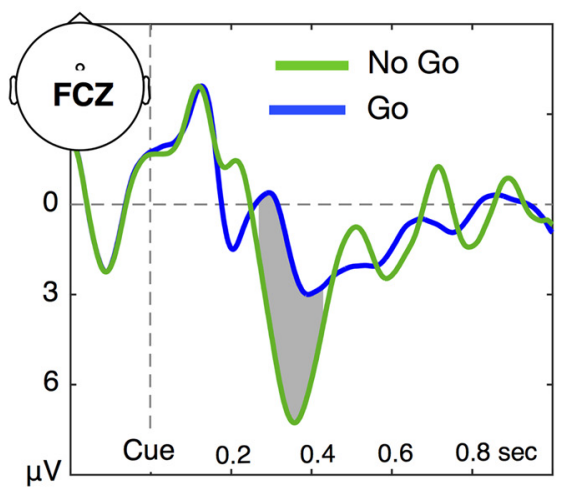

NoGo minus Go

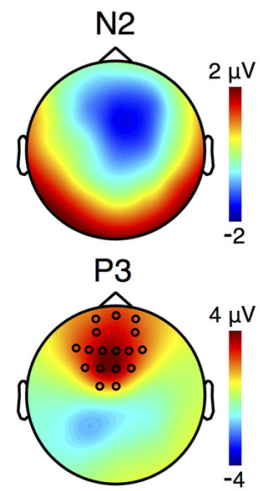

B

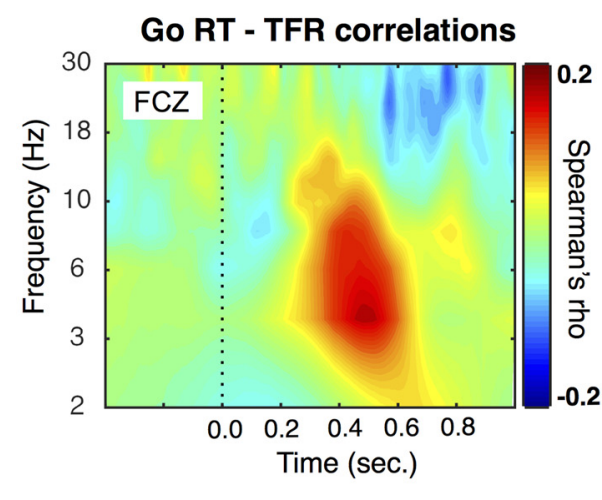

C

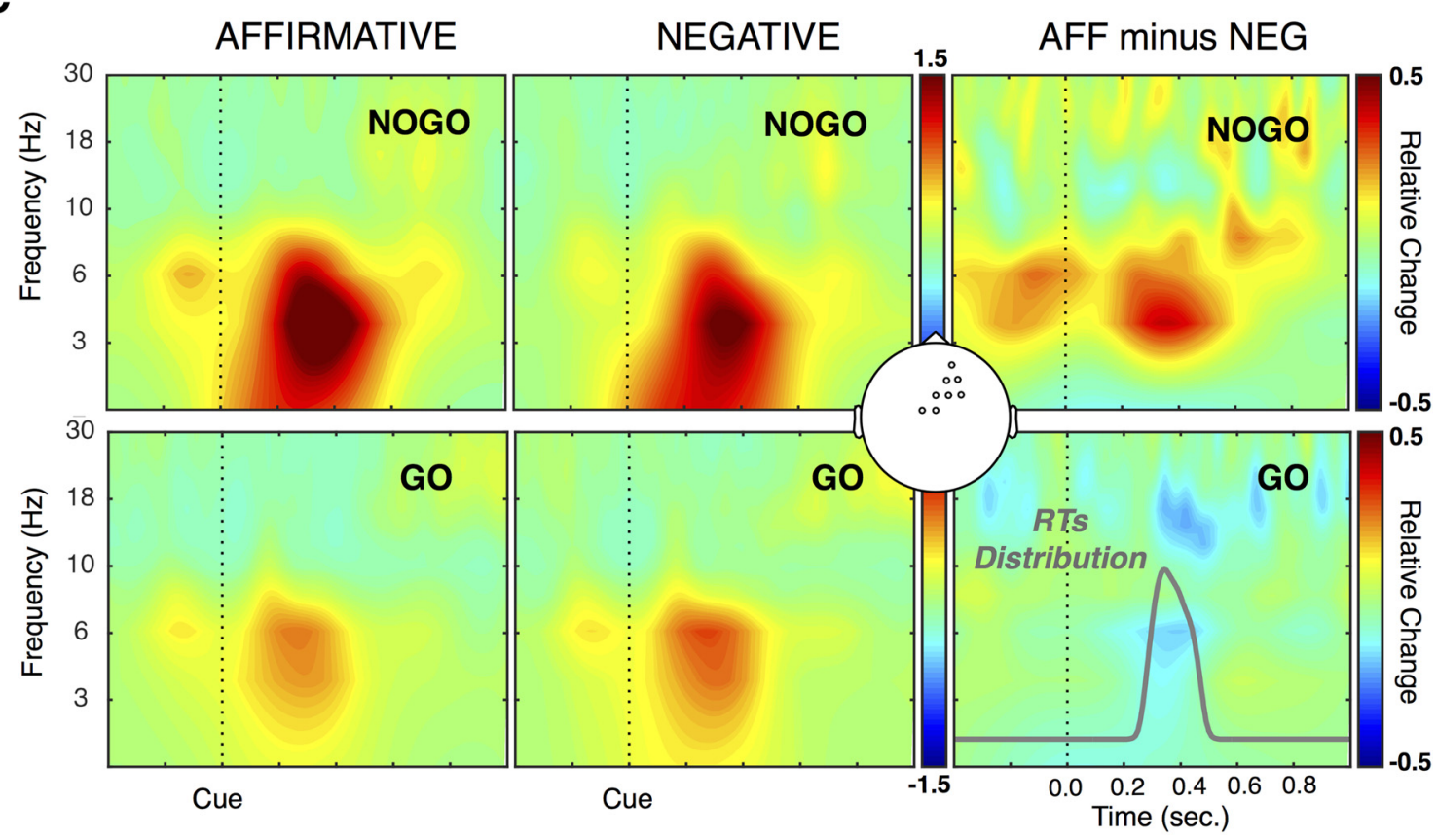

D

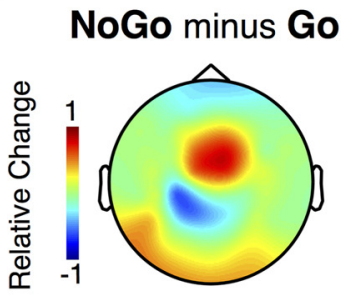

E

AFF minus NEG
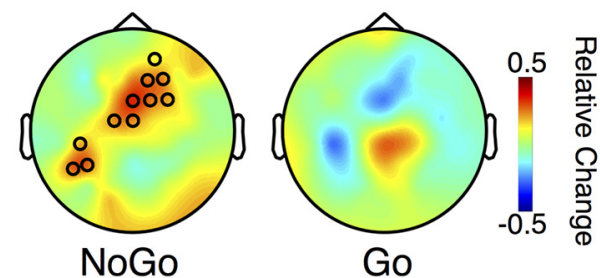

Figure 5. Experiment 2.A, ERP differences between NoGo and Go trials in the $\mathrm{N} 2$ and $\mathrm{P} 3$ components and their distribution on the scalp. $\boldsymbol{B}$, Point-to-point positive correlations between time-frequency EEG signal and reaction times in Go trials for a representative electrode $(\mathrm{FCZ})$. C, Average time-frequency power in the whole set of significant electrodes (signaled in the inserted white map). Affirmative sentences elicited larger theta power than negative sentences in NoGo trials, whereas the pattern reverses in Go trials (cue $\times$ polarity interaction). $\boldsymbol{D}$, Time-frequency analysis of cue main effects: scalp distribution of the significant NoGo-Go differences in theta power. $E$, Topographical distribution of the cue $\times$ polarity interaction in theta-band power $(4-7 \mathrm{~Hz}, 200-550 \mathrm{~ms})$.

In addition, in Experiment 2, there were positive correlations for Go trials between EEG theta power and reaction time around the frequency band $(4-7 \mathrm{~Hz})$ and time window $(200-$ $500 \mathrm{~ms}$.) of the significant TFR cluster (Fig. 5B). Indeed, for a representative electrode of this cluster $(\mathrm{FCz})$, the correlation coefficients showed statistical reliability $\left(t_{(22)}=3.94, p<\right.$
0.001 ), indicating that the larger theta power was the increased amount of time that the response took.

\section{Discussion}

This study presents a new approach to studying the processing of grammatical markers by testing the hypothesis that the compre- 
hension of sentential negations is associated with motor inhibition or conflict monitoring processes in the brain. To this end, participants read affirmative and negative sentences for comprehension while they performed an embedded Go/NoGo task. In the analysis of EEG, we targeted delta- and theta-band oscillations because they could index motor inhibition in the brain. The most important finding obtained in the two experiments was the strong modulation of theta power by sentence polarity. Specifically, for NoGo trials, the increase in frontocentral theta power was larger in the context of affirmative sentences than negative sentences. Therefore, grammatical negation seems to interact with a neurophysiological marker of response inhibition.

The relative reduction of the frontal theta oscillations for NoGo trials in the context of negation could be explained in several ways. First, thetaband modulations could be related to general linguistic processes. An increase of theta power has been associated with lexical-semantic retrieval (Bastiaansen et al., 2005, 2008) and semantic violations in sentence comprehension (Hagoort et al., 2004). However, our theta modulation differs considerably from that of lexical-semantic processes in scalp distribution: frontocentral in this study and temporo-occipital in the aforementioned studies. Moreover, our theta modulation cannot be associated with any distinctive feature of verbs because these were the same across the counterbalanced affirmative and negative sentences. Finally, our theta modulation is an interactive effect occurring during the performance of the $\mathrm{Go} / \mathrm{NoGo}$ task rather than being time locked to the negative operator or the verb onset.

A second possible explanation is that the modulation of theta oscillations relies on the fact that negative sentences are more complex than affirmative sentences, for example, in the number of underlying propositions, steps in mental simulations, etc. The behavioral consequences of the complexity of negated sentences have been reported previously: they slow reading, sentencepicture verification, or probe recognition (for review, see Kaup, 2006). However, it is worth noting that, in the current study, negations did not affect the Go response times; their only behavioral effects were delayed until the sentence recognition task. Moreover, negation did not elicit any main effect on ERP components or oscillatory rhythms, as might be expected if linguistic complexity were the critical factor.

We propose an alternative explanation for the modulatory effects of polarity in the Go/NoGo task observed here: that sentential negation reuses part of the neural mechanisms of response inhibition or action conflict monitoring. Let us focus on the NoGo trials, where the negation operator and the verb preceded the NoGo cue (Fig. 1). We suggest that, in the negative NoGo trials, the negated meaning may be represented in the brain as a degraded stop signal, presetting the response inhibition processes before the NoGo cue appears and consequently reducing the neural inhibitory response (smaller increase in theta power) compared with affirmative-NoGo trials. Conversely, when we consider the effect of polarity in the Go trials, the pattern was reversed. In Experiment 1, larger power in the deltafrequency band $(2-3 \mathrm{~Hz})$ was observed in negative-Go than in affirmative-Go trials, occurring with a larger latency ( $\sim 600 \mathrm{~ms}$ after the $\mathrm{Go} /$ NoGo cue) and with a more central-posterior distribution than the earlier theta effect observed in NoGo trials. In fact, theta and delta could be indexing different functional networks in the brain. Whereas theta could index response conflict processes involved in suppressing a prepotent motor response, delta has been associated with the later evaluation of response processes (Huster et al., 2013; Harper et al., 2014) and error detection (Cohen, 2014). The delta effects of Experiment 1 fit well with this interpretation: inhibition processes are more intense in affirmative NoGo than in negative
NoGo trials as explained above, whereas response evaluation is more intense in negative-Go than in affirmative-Go, because in the former, the system finds a conflict between the executed motor response and the inhibitory presetting associated with the negation meaning. The pattern for Go trials differed in Experiment 2: an increase of theta power for negative-Go trials compared with affirmative-Go trials, rather than the late delta effects obtained in Experiment 1 . We are not sure why the impact of negation on Go trials differs between the two experiments. It could be a consequence of the fact that, in Experiment 2, the critical events (the verb and the $\mathrm{Go} / \mathrm{NoGo}$ cue) were presented faster, which could increase the urgency of the decision and modify the balance of delta- and thetaband oscillatory processes. However, both experiments converge in showing increasing power of one inhibition marker (either theta or delta) in negative-Go trials, which is consistent with the hypothesis that negation presets motor inhibition.

Concerning the behavioral measures, the Go/NoGo task produced a virtual ceiling effect (see low error rate in Experiment 2), so it was not sensitive to the sentence polarity manipulation. Therefore, the presumable relation between performance and neurophysiological measures was not apparent. Moreover, the larger modulation of theta rhythms occurred in NoGo trials, which obviously do not produce motor responses. Despite this, in Experiment 2, we observed a positive correlation between the weak theta-band power variations and response times in Go trials. A possible explanation is as follows: in Go trials, the residual theta activity could index the participants' readiness in not responding (after all, 30\% of trials received a NoGo cue) and, therefore, the larger this preset inhibition is, the more interference it produces with the requested Go response. Interactive effects of cue and polarity were obtained in the recognition task in both experiments: Performance was worse (Experiment 1: slower responses; Experiment 2: increase of errors) in negative-Go trials than in affirmative-Go trials, whereas sentence polarity did not affect recognition in NoGo trials, indicating that the conflict between the preset inhibition in negative sentences and the Go cue has a longterm effect on performance posterior to giving the Go response.

We can speculate that the neural association between negation and response inhibition could have its origins during early language acquisition. In early life, the child is repeatedly exposed to action-verb co-occurrences in everyday manipulative scenarios; for example, in a game-playing setting, the mother could talk about "running the car" while she or the child is running a toy car (Rodrigo et al., unpublished data). These action-verb cooccurrences could contribute, by means of Hebbian-like mechanisms, to the strength of the connections between the perisylvian language regions and the motor and premotor regions (Pulvermüller, 1999, 2013; Glenberg and Gallese, 2012). Moreover, in these early manipulative scenarios, adults frequently use negative imperatives (e.g., "don't touch that") to guide the child's behavior (Wode, 1977; Park, 1979; Austin et al., 2014). In this way, a child could learn the pragmatic function of negative imperatives as stop signals driving him or her to suppress the initiated or intended actions. The pragmatic function of negative imperatives as stop signals may also occur in adults' face-to-face communication. For instance, if a friend tells you "don't drink that!" while you are bringing a drink to your lips, you will probably stop doing it immediately. The co-occurrence of imperative negations and stopping or preventing actions could feed a Hebbian-like mechanism like the one mentioned above, resulting in strengthening the connections between regions of the cortex involved in the lexical representation of negation and those involved in response inhibition or conflict monitoring. This would explain the observed modulation of theta/delta EEG oscillations reported here. 
The present study establishes a new approach to the neurophysiology of linguistic negation, but also has some limitations. The obtained results are constrained to negated actions in imperative form and cannot be generalized to other negated contents. Negative action imperatives are only a subset of the negative polarity sentences used in everyday situations. Many negative sentences involve very abstract contents and have a declarative function. For instance, existential negations, such as "there is no bread" do not refer explicitly to any action, just to the absence of an object. We cannot ensure that the theta modulation observed here with action-related negations also takes place in existential negations. One possibility is that the inhibitory processes marked by frontal theta oscillations are specific to action-related processes (including negated action sentences) and would be absent in existential and other negations not involving actions. However, it is possible that theta-band bursts are a general marker of control or conflict monitoring that could be extended to some cognitive and linguistic processes. If so, then the same theta/delta modulation could be obtained in existential negations and other abstract negations, which also would demand some sort of cognitive conflict resolution. Using the current methodology in future experiments to test these alternatives with different kinds of content could contribute to gaining knowledge about the neurobiology of negation and its relation to control and/or inhibitory processes in the brain.

Meanwhile, we demonstrate here for the first time that the processing of a very abstract syntactic operator such as negation applied to action-related contents interacts with the processing required to suppress a prepotent motor response. The new approach established here is complementary to previous studies demonstrating that negated action sentences reduce the activation of motor processes in the brain compared with affirmative action sentences, yielding "disembodied" representations. However, such studies mainly focus on the consequences of negations on neural representations of sentences, whereas this research offers additional information on the actual processes of negation in the brain. Moreover, this study yields the possibility that the proper neural process of negation is embodied, at least to the extent that motor inhibition is embodied.

\section{References}

Aravena P, Delevoye-Turrell Y, Deprez V, Cheylus A, Paulignan Y, Frak V, Nazir T (2012) Grip force reveals the context sensitivity of language induced motor activity during "action words" processing: evidence from sentential negation. PLoS One 7:e50287. CrossRef Medline

Austin K, Theakston A, Lieven E, Tomasello M (2014) Young children's understanding of denial. Dev Psychol 50:2061-2070. CrossRef Medline

Bartoli E, Tettamanti A, Farronato P, Caporizzo A, Moro A, Gatti R, Perani D, Tettamanti M (2013) The disembodiment effect of negation: negating action-related sentences attenuates their interference on congruent upper limb movements. J Neurophysiol 109:1782-1792. CrossRef Medline

Bastiaansen MC, van der Linden M, Ter Keurs M, Dijkstra T, Hagoort P (2005) Theta responses are involved in lexico-semantic retrieval during language processing. J Cogn Neurosci 17:530-541. CrossRef Medline

Bastiaansen MC, Oostenveld R, Jensen O, Hagoort P (2008) I see what you mean: Theta power increases are involved in the retrieval of lexical semantic information. Brain Lang 106:15-28. CrossRef Medline

Bokura H, Yamaguchi S, Kobayashi S (2001) Electrophysiological correlates for response inhibition in a Go/NoGo task. Clin Neurophysiol 112:2224-2232. CrossRef Medline

Bruin KJ, Wijers AA (2002) Inhibition response mode and stimulus probability: a comparative event-related potential study. Clin Neurophysiol 113:1172-1182. CrossRef Medline

Carpenter PA, Just MA (1975) Sentence comprehension: a psycholinguistic processing model of verification. Psychol Rev 82:45-73. CrossRef
Chambers CD, Garavan H, Bellgrove MA (2009) Insights into the neural basis of response inhibition from cognitive and clinical neuroscience. Neurosci Biobehav Rev 33:631-646. CrossRef Medline

Clark HH, Chase WG (1972) On the process of comparing sentences against pictures. Cogn Psychol 3:472-517. CrossRef

Cohen MX (2014) A neural microcircuit for cognitive conflict detection and signaling. Trends Neurosci 37:480-490. CrossRef Medline

Glenberg AM, Gallese V (2012) Action-based language: A theory of language acquisition comprehension and production. Cortex 48:905-922. CrossRef Medline

Gross J, Kujala J, Hamalainen M, Timmermann L, Schnitzler A Salmelin R (2001) Dynamic imaging of coherent sources: studying neural interactions in the human brain. Proc Natl Acad Sci U S A 98:694-699. CrossRef Medline

Hagoort P, Hald L, Bastiaansen M, Petersson KM (2004) Integration of word meaning and world knowledge in language comprehension. Science 304:438-441. CrossRef Medline

Harper J, Malone S F, Bernat EM (2014) Theta and delta band activity explain N2 and P3 ERP component in go/NoGo task. Clin Neurophysiol $125: .124-132$

Huster RJ, Enriquez-Geppert S, Lavallee CF, Falkenstein M, Herrmann CS, et al. (2013) Electroencephalography of response inhibition tasks: functional networks and cognitive contributions. Int J Psychophysiol 87: 217-233. CrossRef Medline

Kaup B (2001) Negation and its impact on the accessibility of text information. Mem Cognit 29:960-967. Medline

Kaup B (2006) What psycholinguistic negation research tell us about the nature of the working memory representations utilized in language comprehension. Trends Linguist Stud Monographs 173:313-350.

Kaup B, Zwaan RA (2003) Effects of negation and situational presence on the accessibility of text information. J Exp Psychol Learn Mem Cogn 29:439-446. CrossRef Medline

Liuzza MT, Candidi M, Aglioti SM (2011) Do not resonate with actions: Sentence polarity modulates cortico-spinal excitability during actionrelated sentence reading. PLoS One 6:e16855. CrossRef Medline

MacDonald MC, Just MA (1989) Changes in activation levels with negation. J Exp Psychol Learn Mem Cogn 15:633-642. Medline

Maguire MJ, Brier MR, Moore PS, Ferree TC, Ray D, Mostofsky S, Hart J Jr, Kraut MA (2009) The influence of perceptual and semantic categorization on inhibitory processing as measured by the N2-P3 response. Brain Cogn 71:196-203. CrossRef Medline

Maris E, Oostenveld R (2007) Nonparametric statistical testing of EEG- and MEG-data. J Neurosci Methods 164:177-190. CrossRef Medline

Nakata H, Sakamoto K, Ferretti A, Gianni Perrucci M, Del Gratta C, Kakigi R, Luca Romani G (2008) Somato-motor inhibitory processing in humans: an event-related functional. MRI study Neuroimage 39:1858-1866. CrossRef Medline

Nigbur R, Ivanova G, Stürmer B (2011) Theta power as a marker of cognitive interference. Clin Neurophysiol 122:2185-2194. CrossRef Medline

Oostenveld R, Fries P, Maris E, Schoffelen J-M (2011) FieldTrip: open source software for advanced analysis of MEG EEG and invasive electrophysiological data. Comput Intell Neurosci 2011:156869. CrossRef Medline

Park T (1979) Some facts on negation: Wode's four-stage developmental theory of negation revisited. J Child Lang 6:147-151.

Pulvermüller F (1999) Words in the brain's language. Behav Brain Sci 22: 253-336. CrossRef Medline

Pulvermüller F (2013) Semantic embodiment disembodiment or misembodiment? In search of meaning in modules and neuron circuits. Brain Lang 127:86-103. CrossRef Medline

Smith JL, Johnstone SJ, Barry RJ (2008) Movement-related potentials in the Go/NoGo task The P3 reflects both cognitive and motor inhibition. Clin Neurophysiol 119:704-714. CrossRef Medline

Tettamanti M, Manenti R, Della Rosa PA, Falini A, Perani D, Cappa SF, Moro A (2008) Negation in the brain: modulating action representations. Neuroimage 43:358-367. CrossRef Medline

Tomasino B, Weiss PH, Fink GR (2010) To move or not to move: Imperatives modulate action-related motor verb processing in the motor system. Neuroscience 169:246-258. CrossRef Medline

Wode H (1977) Four early stages in the development of L1 negation. J Child Lang 4:87-102. 